\title{
Search for serological markers of infection in transfused patients: are the limitations of these assays always clear?
}

\author{
Massimo De Paschale, ${ }^{1}$ Vittorio Sambri ${ }^{2,3}$ \\ ${ }^{1}$ Microbiology Unit, Hospital of Legnano, ASST Ovest Milanese; ${ }^{2}$ Pievesestina, Microbiology Unit, \\ Romagna Local Health Unit; ${ }^{3}$ DIMES, University of Bologna, Italy
}

Serological investigations for the diagnosis of infection are widely used in the large majority of the Microbiology Laboratories. These assays include the search for antigens and more frequently specific antibodies and are normally used to detect acute, chronic or past infection, depending on the stage of the infection itself and on the hypothesized pathogen. These techniques are mainly applied in the diagnostic workflow for viral infections, but they have also potential usefulness in selected bacterial, fungal and parasitic infections, such as syphilis and toxoplasmosis. Given the wide applicability of these highly automated techniques, their application on samples derived from transfused patients is not uncommon. When used with these particular specimens the results can largely depend on the time between the transfusion and sample collection and on the whole amount of transfused blood components. In detail, these issues may be related to the following two main points: i) the presence of serological markers (typically polyclonal antibodies) in the blood donor, with passive transfer into the transfused patient, that would acquire a different immunological profile depending on the characteristics of the transfused immunoglobulin; ii) the dilution of the patient's whole blood volume generated by the transfusion (this phenomenon could also happen when other fluids are conspicuously introduced in patients, such as when plasma-expanders are used).

The caveat about the limitations in the use of serological assays in transfused patients is thus wide. Despite this fact, clinicians investigating the aetiology of an infection, not always give the due consider-

\author{
Correspondence: Massimo De Paschale, Microbiology Unit, Hospital of Legnano, \\ ASST Ovest Milanese, Via Papa Giovanni Paolo II, 20025 Legnano (MI), Italy. \\ E-mail: massimo.depaschale@asst-ovestmi.it \\ Key words: Serological markers; Infection; Transfused patients. \\ Contributions: the authors contributed equally. \\ Conflict of interest: the authors declare no potential conflict of interest. \\ Received for publication: 10 April 2016. \\ Accepted for publication: 10 April 2016. \\ (C) Copyright M. De Paschale and V. Sambri, 2016 \\ Licensee PAGEPress, Italy \\ Microbiologia Medica 2016; 31:6325 \\ doi: $10.4081 / \mathrm{mm} .2016 .6325$
}

This article is distributed under the terms of the Creative Commons Attribution Noncommercial License (by-nc 4.0) which permits any noncommercial use, distribution, and reproduction in any medium, provided the original author(s) and source are credited. ation to this problem that could generate clinical misinterpretation of the serological data. In addition, the information about the recent injection of blood related biological products via transfusion is often not made available to the Microbiology Laboratory.

As biological markers of infection, in most of the cases specific IgG and IgM and, much less frequently, IgA, are sought. For selected stages of some viral and fungal infections, plasma circulating antigens can also be directly investigated, such as in the case of Hepatitis B surface antigen (HBsAg) detection for hepatitis B virus (HBV) infection. Since all blood-donated units are routinely screened for the presence of HBsAg and only negative the ones are allowed to be used for transfusion practices, the identification of this marker in a transfused patient is undoubtedly an index of active HBV infection since its transmission via transfusion is highly unlike. The same behaviour is demonstrated for anti-hepatitis C virus, anti-HIV or syphilis antibodies since all blood products are screened for these infections. This is not the case for the detection of anti-HBs (that is HBsAb). Blood components are allowed to be taken from donors vaccinated against HBV (and therefore antiHBs positive) or from donors with $\mathrm{HBV}$ past infection [in this case the serological profile would be anti-HBs and core antigen of hepatitis B virus (anti-HBc) positive]. As a consequence, the detection of anti$\mathrm{HBs}$ and anti-HBc in a transfused patient could either be the result of the patient's immune response against HBV or the passive transfer of these specific antibodies by transfusion (this is more likely when these anti-HBV specific antibodies are detected at a low titre). In this latter case, the results of serological testing can be clinically misinterpreted (of course when no information about the transfusion status of the patient is provided).

The level of awareness about this possible clinical misinterpretation of serological data is quite high for inpatients that could be transfused many times during their hospital stay. On the other hand, this phenomenon is likely underestimated for outpatients or for those subjects that are moving from one clinical department to another (or when the patients are transferred to a different care giving setting) being the above conditions a well-known cause of failures in the information transfer about the occurrence and timing of blood transfusion.

For other markers of infection not routinely screened on blood donations, the situation is even more problematic because it is difficult to determine the presence of specific antibody in the blood product (1). For the diagnosis of many different infections antigen specific IgG and IgM are normally sought. The presence of IgM against a specific infectious agent is very unlikely (even not completely impossible) in a blood donation since a clinically evident infection is a status that makes the donation itself not acceptable by the Blood bank system. This could indeed happen in the case of blood donation in the asymptomatic or pre-symptomatic stage of infection. The detection of IgG in the absence of IgM is normally interpreted as a marker of past infection and do not have clinical relevance. This can anyway give problems of interpretation of the serological data in the cases of serial samples 
obtained from (poly)transfused patients when IgG are variably detected in one samples from another. In these patients it is of outmost relevance to investigate the presence of the same anti-antigen specific IgG in the donor. Furthermore, in some (limited) cases, the IgM immune response is not detected (or with a very low titre), so the acute stage of infection is detectable only by the presence of specific IgG seroconversion $(6,7)$. In this case it is difficult to understand if the presence of IgG is actually generated by a post infection seroconversion or by passive transfer following transfusion. No doubt that in these cases other microbiological investigations must be carried out: in addition to search for the presence of IgG in the donor (which may not be diriment or easy to be performed), the detection of pathogen nucleic acids by molecular biology techniques in transfused patient can help solving the problem (1). Being the issue clear and in case of strong clinical suspicion, clinicians should not only rely their diagnosis on serology testing but should prescribe, when available, pathogen specific molecular biology tests. This must also be considered (and strongly suggested to the Clinicians) by the Microbiology Laboratory, in particular in all the cases of inconsistent antibody detection in serial serum samples. Furthermore, a clinical microbiology comment placed in the laboratory report such as the immune response is indicating a likely past infection (obvious in the case of detection of IgG without IgM), can be confusing. This kind of comment is perfectly suitable for not transfused patient, but in transfused patients this sentence could lead the clinician to a wrong diagnosis. This is even more likely when the report is reviewed after some time.

In the overall clinical evaluation of the serological report in transfused patients it is important to keep in mind the time interval between the transfusion and the collection of the serum sample for the microbiological diagnosis (the shelf-life of passively antibodies introduced with transfusion, estimated in about 25 days, should be considered as well) (3). In addition it is important to critically evaluate the number of transfusions (and the interval between the different transfusions) received by each individual patients. It is also relevant to consider the type of transfused blood component, since the passive transfer of IgG, can vary depending on the different type of blood component (packed red blood cells, platelets or plasma) used. The initial antibody titre of donors, whose blood components are assembled into pools (for example in the platelet pool), is another determining factor. Most of these variables are difficult to verify, and for this reason the use of molecular biology tests can be helpful. It is also relevant to state a clear and factitive cooperation between Clinical Microbiologists and the Blood Bank systems.

The transfusion of whole blood or blood components, however, is not only confusing for the antibody presence in the donor, but also for the dilution effect that this therapeutic intervention generate into the receiving patients. A massive blood transfusion (as well as for the infusion of large volume of plasma-expanders substances) can lead to a clinical false negative detection of pathogen specific antibodies. For example, the negativity of HBsAg after an abundant transfusion of plasma can be non reliable for the exclusion of an acute HBV infection. The guidelines issued by the Italian National Transplant Centre for the serological testing of tissue donors, indicate that if the donor sample dilution, due to transfusion or infusion of plasma expanders in the 48 hours before withdrawal, is more than $50 \%$, the result is not reliable and the donor must not considered suitable for donation. To ascertain the donor suitability, when the dilution is hypothetically low, all the necessary parameters must however be given for the calculation of the dilution level, but it is more appropriate and preferable to perform the serology testing in pre-transfusion obtained samples, when available. In this case the pre-transfusion sample are generally available from the Blood and Organ Bank system where they are stored after the immune typing and tissue compatibility screening procedure (4). The effect of the massive dilution is therefore decisive in evaluating the results (moreover also normal blood sample should not be run near the point of entry of an infusion), but in the case of clinical suspicion even lower dilutions should be kept in mind as the marker may have been present at very low level in the patient before the transfusion. The repetition of the test after some time from transfusions (if clinically possible) can help to improve the evaluation of these data.

In conclusion, the serology testing in transfused patients has always been known as a problematic diagnostic procedure. This could also be the problem in samples obtained from patients receiving massive doses of therapeutic immunoglobulins) $(2,5)$. Consequently, an accurate transfer of all the available information and a close cooperation between microbiologists, Blood Banks doctors and clinicians are vital for the correct evaluation of the serology tests in this potentially problematic population of patients.

\section{References}

1. Bélanger SS, Fish D, Kim J, Cohen S. False-positive human T-lymphotropic virus serology after intravenous immunoglobulin transfusion. CMAJ 2012;184:1709-12.

2. Lichtiger B. Laboratory serologic problems associated with administration of intravenous IgG. Curr iss Transf Med 1994;3.

3. Mankarious S, Lee M, Fischer S, et al. The half-lives of IgG subclasses and specific antibodies in patients with primary immunodeficiency who are receiving intravenously administered immunoglobulin. J Lab Clin Med 1988;112:634-40.

4. Ministero della salute. Linee guida del Centro Nazionale Trapianti - Linee guida per il prelievo, la processazione e la distribuzione di tessuti a scopo di trapianto. LG 19060710. Luglio 2013.

5. Pelloux H, Fricker-Hidalgo H, Brochier G, et al. Intravenous immunoglobulin therapy: confounding effects on serological screening for toxoplasmosis during pregnancy. J Clin Microbiol 1999;37:3423-4.

6. Schillinger M, Kampmann M, Henninger K, et al. Variability of humoral immune response to acute Epstein-Barr virus (EBV) infection: evaluation of the significance of serological markers. Med Microbiol Lett 1993;2:296-303.

7. Schmid DS, Jumaan AO. Impact of varicella vaccine on varicellazoster virus dynamics. Clin Microbiol Rev 2010;23:202-17. 